\section{Cold fusion doubts and controls}

SIR-Much premature excitement has been generated by the claims and counterclaims for induced cold fusion. There is apparently no consensus (Nature 338, 616; 1989 ) on the central questions regarding the amount of excess heat produced, the evidence for nuclear reactions and the vexed question of whether the detection of reaction fragments could be consistent with the claims for excess heat generation.

For many years we have been investigating primordial nucleosynthesis in a hot Big-Bang cosmology. The measured abundances of the light isotopes of hydrogen, helium and lithium place stringent restrictions on a number of physical parameters and are sensitive to deuteriumdeuterium reaction rates. We have investigated by how much these reaction rates can be increased by a single low-energy resonance, consistent with deuteriumdeuterium laboratory data, without destroying the ability to provide a consistent description of primordial lightisotope abundances.

Armed with these maximum reaction rates, we can estimate the density of deuterium packing in the electrodes of one of the cold-fusion experiments in order to generate excess heat at the rate of watts, as reported in some experiments. Our result is $10^{7} \mathrm{~mol} \mathrm{~cm}^{-3}$. We conclude that more heat has been generated in the media coverage of this subject than by deuterium fusion in electrolytic cells.

J. M. IRVINE

S. RILEY

Department of Theoretical Physics,

Schuster Laboratory,

The University,

Manchester M13 9PL, UK

SIR-Fleischmann, Pons and Hawkins' claimed' detection of neutrons and tritium from a postulated $d-d$ reaction is not yet supported by their account of the controls used to eliminate other sources of both neutrons and tritium. The control on neutron background reported in ref. 1 - a series of measurement with the deuteriumcontaining cell removed - is no control on neutrons arising from photodisintegration of deuterons or other materials in the cell by ambient $\gamma$-rays. Such a background could also be a problem with the reported neutron flux of Jones et al. ${ }^{2}$. A check on that background would be to determine whether the neutrons appeared and disappeared when the power to the electrochemical cell was switched on and off.

The tritium measurement is even more problematic; one must have controls against the possibility of neutron capture creating tritium by the reactions $\mathrm{D}(\mathrm{n}, \gamma) \mathrm{T}$ and ${ }^{6} \mathrm{Li}(\mathrm{n}, \alpha) \mathrm{T}$. Because tritium decay cannot be turned off, supplementary NATURE · VOL 339 - 15 JUNE 1989 experiments may be needed to show that tritium does not grow into a vessel freshly filled with tritium-free $\mathrm{D}$ and ${ }^{6} \mathrm{Li}$ by either of the postulated reactions. Tritium is also known to exchange quickly with other hydrogen isotopes, is widely used in commerce $^{3}$, and is a durable contaminant of laboratories which have been exposed to tritium in various forms.

Note that repetition of the experiments in refs 1 and 2 with deuterium replaced by hydrogen is not a control on the suggested sources of neutrons or tritium. A negative result with tritium-free hydrogen is therefore moot with respect to claims of cold fusion based on neutron or tritium detection in a $\mathrm{D}^{-6} \mathrm{Li}$ mixture.

LAWRENCE CRANBERG

TDN Inc., Austin,

Texas 78764, USA

1. Fleischmann, M., Pons, B. \& Hawkins, M. J. electroanalyt. Chem. 261, 301-308 (1989)

2. Jones, S.E. et al. Nature 338, 737-740 (1989).

3. Moghissi, A.A. et al. (eds) Radioactivity in Consumer Pro ducts (US Nuclear Regulatory Commission, Washington, DC, 1978)

\section{Radiolysis of water}

SIR-Baverstock and Cundall' suggest that the energy imparted directly and locally by ionizing radiation to a DNA molecule may manifest itself as broken bonds at a distance because, between the time of energy deposition and bond breaking, the energy is converted to a soliton, which may comprise a wave of coupled vibrational and electronic excitation travelling along the molecule.

In an electron-nuclear double-resonance study ${ }^{2}$ of the crystalline malonic acid-urea 1:1 adduct (again a hydrogen-bonded structure) irradiated at room temperature, a signal was observed which was ascribed to a solitonic wave. This wave is excited and sustained by the presence of doublewell hydrogen bonds in the crystal. The crystal structure consists of planar layers held together by a complex network of hydrogen bonds within the bond planes.

If we extend these ideas from the oneand two-dimensional structures above to a three-dimensional case, the classic example of such a hydrogen-bonded structure is liquid water. The initial behaviour of water irradiated at room temperature exhibits certain anomalies ${ }^{3}$, which defy explanation if we base our ideas about energy deposition from high-energy radiation on gas-phase interactions, because this approach takes insufficient account of liquid structure.

In particular, in the $a b$ initio modelling of product yields, the stochastic method of kinetic calculations ${ }^{4}$, when used in conjunction with a well-known method of deriving the distribution ${ }^{5}$ in spur energy, requires (in an acid system) the unit radius for $\mathrm{H}$ or $\mathrm{e}_{\mathrm{a}}^{-}$to be as low as $0.44 \mathrm{~nm}$ and to be $0.19 \mathrm{~nm}$ for $\mathrm{OH}$, in order to obtain the observed results for $G\left(\mathrm{H}_{2}\right)$ and $G\left(\mathrm{H}_{2} \mathrm{O}_{2}\right)$ (where $G(\mathrm{M})$ is the number of molecules of $\mathrm{M}$ formed per $100 \mathrm{eV}$ of radiation energy absorbed). In contrast, the deterministic method requires more credible values of 2.3 and $0.75 \mathrm{~nm}$, respectively. This is partly because, for the more abundant smaller spur sizes, the stochastic method gives a greater ratio of combination for unlike relative to like pairs of radicals.

This difficulty would be mainly obviated if the typical entity decomposed by radiation were a group of five to six molecules", part of the 'flickering cluster' structure of liquid water ${ }^{7}$, in which the secondary electrons in the lower part of the energy distribution (below, say, $50 \mathrm{eV}$ ) give rise to a solitonic wave whose energy could be dissipated in these clusters in nondissociative processes, but in which higher electron energies would cause dissociation and ionization. Such a model would remove the proportionality previously assumed between the energy per spur and the number of ionizations per spur (taken, for example, to be 6 ionizations per $100 \mathrm{eV}$ in ref. 5, or 12 radicals per $100 \mathrm{eV}$ in ref. 4), irrespective of the amount of energy imparted. To obtain the correct average ionization yield (about one ion pair per $20 \mathrm{eV}$ ), the energy transferred in packets larger than a threshold level of about $50 \mathrm{eV}$ would need to be more efficient in producing ionizations, but the use of this modified distribution in the number of ionizations per spur would then give results close to those for a set of uniform spurs of 5-6 ionizations per spur, for which the stochastic and deterministic models give similar results ${ }^{*}$. In addition, the resulting, more realistic, unit spur radii of $2.3 \mathrm{~nm}$ for aquated electrons and $0.75 \mathrm{~nm}$ for hydroxyl radicals would give rise to agreement between prediction and observation in the effects of scavengers and of linear energy transfer, and also in time-resolved studies ${ }^{9,10}$ as there is reasonable agreement in all these areas when the deterministic method is used ${ }^{3.6}$.

An alternative stochastic approach ${ }^{11}$ is showing interesting possibilities in some of these areas, especially in predicting scavenger effects at early times and timeresolved yields of $\mathrm{e}_{\mathrm{aq}}^{-}$for fast electrons ${ }^{4}$ and for $3-\mathrm{MeV}$ protons ${ }^{10}$ - but the role of normalization at $10 \mathrm{ps}$ in the ab initio calculation of ref. 11 was not adequately discussed.

In cases where the first-order structure of the water is broken down, such as during high-temperature $\left(300{ }^{\circ} \mathrm{C}\right)$ $\gamma$-radiolysis (A.J. Elliot et al., personal communication) or for densely ionizing radiation at room temperature ${ }^{12}$, more product formation is observed than can be accounted for by normal diffusioncontrolled kinetics. This may be because the lower-energy secondary electrons 